\title{
Multifocal motor neuropathy in idiopathic non-cirrhotic portal hypertension
}

\author{
Santosh Laxman Wakode ${ }^{1}$, Naveen Ravi ${ }^{1}$, Amit Agrawal ${ }^{2}$ \\ ${ }^{1}$ Department of Physiology, AlIMS, Bhopal, India \\ ${ }^{2}$ Department of Neurosurgery, AlIMS, Bhopal, India
}

Multifocal motor neuropathy (MMN) is characterized by progressive asymmetrical motor weakness of distal limbs without sensory involvement. It is a rare immunological disorder $(1,2)$. Hallmark features of MMN on nerve conduction study (NCS) include motor nerve conduction blocks (CB) with normal sensory nerve action potentials (SNAP) $(1,2)$. Idiopathic non-cirrhotic portal hypertension (INC-PH) is relatively commoner and is characterized by features of $\mathrm{PH}$ in the absence of cirrhosis or other causes of liver disease. Immunological factors have been linked to $\operatorname{INCPH}(3,4)$. However, till date, to the best of our knowledge there have been no reports of MMN in INCPH. A47-year-old female presented with the history of esophageal varices 7 years ago. Following investigations, she was diagnosed with INCPH and received treatment for the same. Ever since, she has been on follow up with periodic liver function tests (LFT) and ultrasound (USG) abdomen. LFT ranged from being normal to mildly deranged. USG consistently demonstrated features of splenomegaly, presence of collaterals, and dilated portal vein. Since the last three months, she noticed gradually worsening weakness of both upper limbs and right lower limb, without any sensory disturbances. From one month she developed bilateral clawing of hands, more pronounced in left hand. Motor NCS findings revealed prolonged distal latencies

Conflict of interest: none declared

Financial support: none declared of left median nerve (MN) and ulnar nerve (UN), on distal stimulation (figures 1A,C). Normal compound muscle action potentials were recorded from right $\mathrm{MN}, \mathrm{UN}$ and peroneal nerve $(\mathrm{PN})$ on distal stimulation (figures $1 \mathrm{~F}, \mathrm{H}, \mathrm{E}$ ). There were $\mathrm{CB}$ on proximal stimulation of bilateral $\mathrm{MN}, \mathrm{UN}$ and right PN (figures 1B,G,D,I,J). Normal SNAP were recordable from bilateral $\mathrm{MN}, \mathrm{UN}$ and right sural nerves. NCS features were pathognomonic of MMN. She declined cerebrospinal fluid analysis and titers of anti GM1 IgM antibodies. She was subsequently treated with intravenous immunoglobulins (IVIG), following which there was a drastic improvement in her motor symptoms. However, USG and LFT were similar to those obtained prior to IVIG therapy. Diagnosis of MMN requires high degree of suspicion and correlation with NCS can assist in it. Awareness that MMN can occur in INCPH can help facilitate broadening the horizon of clinicians and in opening up new possibilities for further research. To the best of our knowledge, this is the first study reporting features of MMN in INCPH. However, based on the findings of present study it was not possible to propose if this association was causal or sporadic.

Keywords: multifocal motor neuropathy, idiopathic non-cirrhotic portal hypertension, nerve conduction study 


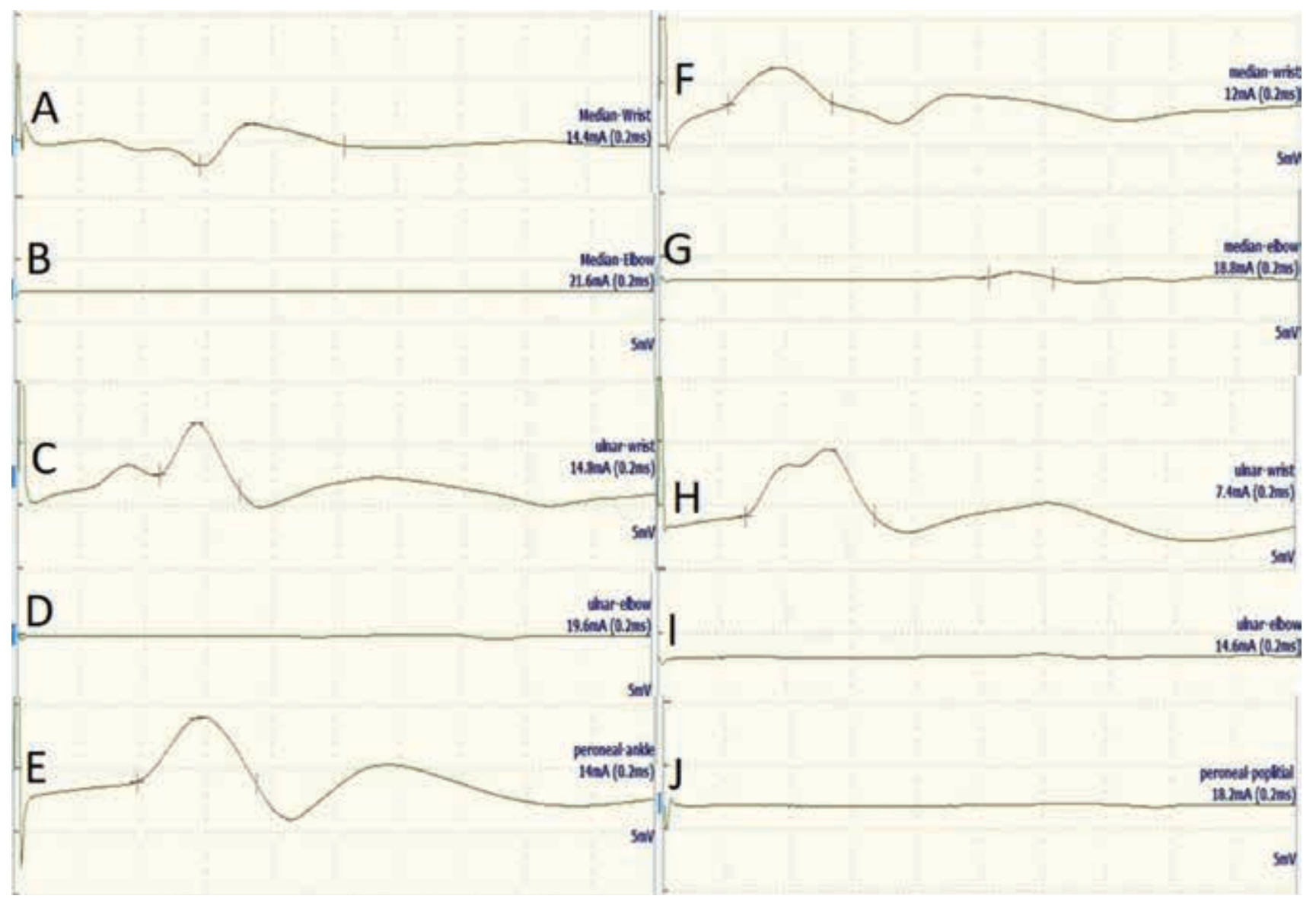

FIGURE 1. Motor nerve conduction study waveforms. A and B - Left median nerve, distal and proximal stimulation respectively, while recording over abductor pollicis brevis (APB). C and D - Left ulnar nerve, distal and proximal stimulation while recording over abductor digiti minini (ADM). E - Right peroneal nerve, distal stimulation while recording over extensor digitorum brevis (EDB). F and $G$ - Right median nerve, distal and proximal stimulation, while recording over $A P B$. $H$ and I - Right ulnar nerve, distal and proximal stimulation while recording over ADM. $J$-Right peroneal nerve on proximal stimulation while recording over EDB

TABLE 1. Motor NCS CAMP values

\begin{tabular}{|l|c|c|c|c|}
\hline Nerve & Side & Site & $\begin{array}{c}\text { Latency } \\
(\mathbf{m s})\end{array}$ & $\begin{array}{c}\text { Amplitude } \\
(\mathbf{m V})\end{array}$ \\
\hline Median & Left & Distal & 8.7 & 3.3 \\
\hline Median & Left & Proximal & - & - \\
\hline Ulnar & Left & Distal & 6.7 & 4.1 \\
\hline Ulnar & Left & Proximal & - & - \\
\hline Median & Right & Distal & 3.3 & 2.9 \\
\hline Median & Right & Proximal & 15.4 & 0.4 \\
\hline Ulnar & Right & Distal & 4.1 & 5.2 \\
\hline Ulnar & Right & Proximal & - & - \\
\hline Peroneal & Right & Distal & 5.8 & 5 \\
\hline Peroneal & Right & Proximal & - & - \\
\hline
\end{tabular}

\section{REFERENCES}

1. Vlam L, van der Pol W, Cats E, et al. Multifocal motor neuropathy: diagnosis, pathogenesis and treatment strategies. Nature Reviews Neurology. 2011;8(1):48-58.

2. Yeh WZ, Dyck PJ, van den Berg LH, et al. Multifocal motor neuropathy: controversies and priorities. J Neurol Neurosurg Psychiatry. 2020;91:140-148.

3. Schouten J, Verheij J, Seijo S. Idiopathic non-cirrhotic portal hypertension: a review. Orphanet J Rare Dis. 2015;10(1).

4. Guido M, Sarcognato S, Sacchi D, Colloredo G. Pathology of idiopathic non-cirrhotic portal hypertension. Virchows Archiv. 2018;473(1):23-31. 